\title{
Analytic Solutions of the Space-Time Fractional Combined KdV-mKdV Equation
}

\author{
Emad A.-B. Abdel-Salam ${ }^{1,2}$ and Zeid I. A. Al-Muhiameed ${ }^{3}$ \\ ${ }^{1}$ Department of Mathematics, Faculty of Science, Assiut University, New Valley Branch, El-Kharja 72511, Egypt \\ ${ }^{2}$ Department of Mathematics, Faculty of Science, Northern Border University, Arar 91431, Saudi Arabia \\ ${ }^{3}$ Department of Mathematics, Faculty of Science, Qassim University, Buraydah 51452, Saudi Arabia
}

Correspondence should be addressed to Emad A.-B. Abdel-Salam; emad_abdelsalam@yahoo.com

Received 27 May 2014; Revised 25 August 2014; Accepted 26 August 2014

Academic Editor: Zhong-Ke Gao

Copyright (C) 2015 E. A.-B. Abdel-Salam and Z. I. A. Al-Muhiameed. This is an open access article distributed under the Creative Commons Attribution License, which permits unrestricted use, distribution, and reproduction in any medium, provided the original work is properly cited.

\begin{abstract}
The fractional mapping method is proposed to solve fractional differential equations. To illustrate the effectiveness of the method, we discuss the space-time fractional combined KdV-mKdV equation. Many types of exact analytical solutions are obtained. The solutions include generalized trigonometric and hyperbolic functions solutions. These solutions are useful to understand the mechanisms of the complicated nonlinear physical phenomena and fractional differential equations. Among these solutions, some are found for the first time.
\end{abstract}

\section{Introduction}

In the last two decades, a large class of fractional differential equations (FDEs) have been derived and widely applied in various branches of natural science such as chemistry, biology, mathematics, communication, and particularly in almost all branches of physics. Numerical and analytical solutions of FDEs play an important role in nonlinear phenomena; especially in nonlinear physical problems since they can provide much physical information, thus leading to further applications. Seeking the numerical and analytical solutions of FDEs has long been an interesting and hot topic in the nonlinear mathematical physics [1-7]. Many effective methods for obtaining numerical and analytical solutions of FDEs have been presented such as finite difference method $[8,9]$, finite element method [10], Adomian decomposition method [11, 12], differential transform method [13], variational iteration method [14-16], homotopy perturbation method [17, 18], spectral methods $[19,20]$, discontinuous Galerkin method [21], Kansa method [22], the fractional subequation method [23], and generalized fractional subequation method [24]. Abdel-Salam and Yousif [25] introduced the fractional Riccati expansion method to obtain analytical solutions of FDEs with constant coefficients. They solved the space-time fractional $\mathrm{KdV}$ equation, regularized long-wave equation, Boussinesq equation, and Klein-Gordon equation. In this research paper, we introduce the fractional mapping method to obtain many exact traveling wave solutions of nonlinear FDEs with the Jumarie's modified Riemann-Liouville derivative [26-28]. We apply the considered method to solve the space-time fractional combined KdV-mKdV equation.

The structure of this paper is as follows: some basic definitions of the fractional calculus and the description of the fractional mapping method are introduced in Section 2. In Section 3, we apply the fractional mapping method for solving the space-time fractional combined KdV-mKdV equation. Finally, we discuss the findings and conclude.

\section{Description of the Fractional Mapping Method}

In this section we present the fractional mapping method to construct exact analytical solutions of nonlinear FDEs 
with the modified Riemann-Liouville derivative defined by Jumarie [26-28]:

$$
\begin{aligned}
& D_{x}^{\alpha} f(x) \\
& \quad=\left\{\begin{array}{lc}
\frac{1}{\Gamma(1-\alpha)} \int_{0}^{x}(x-\xi)^{-\alpha-1}[f(\xi)-f(0)] d \xi \\
\frac{1}{\Gamma(1-\alpha)} \frac{d}{d x} \int_{0}^{x}(x-\xi)^{-\alpha}[f(\xi)-f(0)] d \xi \\
{\left[f^{(\alpha-n)}(x)\right]^{(n)},} & 0<\alpha<1
\end{array}\right. \\
&
\end{aligned}
$$

which has merits over the original one, for example, the $\alpha$ order derivative of a constant is zero. Some properties of the Jumarie's modified Riemann-Liouville derivative are

$$
\begin{gathered}
D_{x}^{\alpha} x^{\gamma}=\frac{\Gamma(\gamma+1)}{\Gamma(\gamma+1-\alpha)} x^{\gamma-\alpha}, \quad \gamma>0 \\
D_{x}^{\alpha}(c f(x))=c D_{x}^{\alpha} f(x), \\
D_{x}^{\alpha}[f(x) g(x)]=g(x) D_{x}^{\alpha} f(x)+f(x) D_{x}^{\alpha} g(x), \\
D_{x}^{\alpha} f[g(x)]=f_{g}^{\prime}[g(x)] D_{x}^{\alpha} g(x)=D_{g}^{\alpha} f[g(x)]\left(g_{x}^{\prime}\right)^{\alpha},
\end{gathered}
$$

where $c$ is constant. The formulas (4) and (5) follow from the fractional Leibniz rule and the fractional Barrow's formula [29]. In addition, Kolwankar obtained the same formula (4) by using an approach on Cantor space [30]. In addition, Jumarie in [31] gave detailed proofs of the above formulas (see Proposition 3.1 page 1746 and Section 4 (Some Basic Formulae for Fractional Derivative and Integral) page 1748). The above properties play an important role in the fractional mapping method. The main steps of this method are described as follows.

Step 1. Suppose that the nonlinear FDE, say in two variables $x$ and $t$, is given by

$$
P\left(u, D_{t}^{\alpha} u, D_{x}^{\alpha} u, D_{t}^{2 \alpha} u, D_{x}^{2 \alpha} u, \ldots\right)=0, \quad 0<\alpha \leq 1
$$

where $D_{t}^{\alpha} u$ and $D_{x}^{\alpha} u$ are Jumarie's modified RiemannLiouville derivatives of $u, u=u(x, t)$ is an unknown function, $P$ is a polynomial in $u$, and its various partial derivatives.

Step 2. By using the traveling wave transformation

$$
u(x, t)=u(\xi), \quad \xi=k x+\omega t,
$$

where $\omega$ and $k$ are constants to be determined later. The nonlinear FDE (6) is reduced to the following nonlinear fractional ordinary differential equation (FODE) for $u(x, t)=$ $u(\xi)$ :

$$
\widetilde{P}\left(u, \omega^{\alpha} D_{\xi}^{\alpha} u, k^{\alpha} D_{\xi}^{\alpha} u, \omega^{2 \alpha} D_{\xi}^{2 \alpha} u, k^{2 \alpha} D_{\xi}^{2 \alpha} u, \ldots\right)=0 .
$$

Step 3. We assume that $u(\xi)$ can be expressed by a finite power series of $F(\xi)$ :

$$
u(\xi)=a_{0}+\sum_{i=1}^{n} a_{i} F^{i}, \quad a_{n} \neq 0
$$

where $a_{i}(i=0,1,2, \ldots, n)$ are constants to be determined later, $n$ is a positive integer determined by balancing the linear term of the highest order with the nonlinear term in (8) and $F=F(\xi)$ satisfies the fractional elliptic equation:

$$
D_{\xi}^{\alpha} F=\sqrt{A+B F^{2}+C F^{4}}, \quad 0<\alpha \leq 1,
$$

where $A, B$, and $C$ are constants. Using the Mittag-Leffler function in one parameter $E_{\alpha}(x)=\sum_{\ell=0}^{\infty}\left(x^{\ell} / \Gamma(1+\alpha \ell)\right)(\alpha>$ $0)$, (10) has different solutions, so we ought to discuss solution of (10) in the following cases (detailed proof of these cases is in the appendix).

Case 1. If $A=1, B=-2$, and $C=1$, then (10) has the following solution:

$$
F=\tanh (\xi, \alpha), \quad F=\operatorname{coth}(\xi, \alpha)
$$

Case 2. If $A=0, B=1$, and $C=-1$, then (10) has the solution $F=\operatorname{sech}(\xi, \alpha)$.

Case 3. If $A=1 / 4, B=-1 / 2$, and $C=1 / 4$, then (10) has the following solution:

$$
\begin{gathered}
F=\frac{\tanh (\xi, \alpha)}{1 \pm \operatorname{sech}(\xi, \alpha)}, \\
F=\operatorname{coth}(\xi, \alpha)+\operatorname{csch}(\xi, \alpha), \\
F=\tanh (\xi, \alpha)+i \operatorname{sech}(\xi, \alpha), \quad i=\sqrt{-1}
\end{gathered}
$$

Case 4. If $A=0, B=1$, and $C=1$ then (10) has the solution $F=\operatorname{csch}(\xi, \alpha)$.

Case 5. If $A=1, B=1$, and $C=0$, then (10) has the solution $F=\sinh (\xi, \alpha)$.

Case 6. If $A=-1, B=1$, and $C=0$, then (10) has the solution $F=\cosh (\xi, \alpha)$.

Case 7. If $A=1, B=-1$, and $C=0$, then (10) has the solution

$$
F=\cos (\xi, \alpha), \quad F=\sin (\xi, \alpha)
$$

Case 8. If $A=0, B=-1$, and $C=1$, then (10) has the solution

$$
F=\sec (\xi, \alpha), \quad F=\csc (\xi, \alpha) .
$$

Case 9. If $A=1, B=2$, and $C=1$, then (10) has the solution

$$
F=\tan (\xi, \alpha), \quad F=\cot (\xi, \alpha)
$$


where the generalized hyperbolic and trigonometric functions are defined as

$$
\begin{gathered}
\cosh (\xi, \alpha)=\frac{E_{\alpha}\left(\xi^{\alpha}\right)+E_{\alpha}\left(-\xi^{\alpha}\right)}{2}, \\
\sinh (\xi, \alpha)=\frac{E_{\alpha}\left(\xi^{\alpha}\right)-E_{\alpha}\left(-\xi^{\alpha}\right)}{2}, \\
\cos (\xi, \alpha)=\frac{E_{\alpha}\left(i \xi^{\alpha}\right)+E_{\alpha}\left(-i \xi^{\alpha}\right)}{2}, \\
\sin (\xi, \alpha)=\frac{E_{\alpha}\left(i \xi^{\alpha}\right)-E_{\alpha}\left(-i \xi^{\alpha}\right)}{2 i}, \\
\tanh (\xi, \alpha)=\frac{\sinh (\xi, \alpha)}{\cosh (\xi, \alpha)}, \quad \tan (\xi, \alpha)=\frac{\sin (\xi, \alpha)}{\cos (\xi, \alpha)}, \\
\operatorname{coth}(\xi, \alpha)=\frac{1}{\tanh (\xi, \alpha)}, \quad \operatorname{sech}(\xi, \alpha)=\frac{1}{\cosh (\xi, \alpha)}, \\
\cot (\xi, \alpha)=\frac{1}{\tan (\xi, \alpha)}, \quad \sec (\xi, \alpha)=\frac{1}{\cos (\xi, \alpha)}, \\
\operatorname{csch}(\xi, \alpha)=\frac{1}{\sinh (\xi, \alpha)}, \quad \csc (\xi, \alpha)=\frac{1}{\sin (\xi, \alpha)} .
\end{gathered}
$$

Step 4. Substituting (9) into the FODE (8) and taking into account (10), the left-hand side of (8) can be converted into a polynomial in $F(\xi)$. Equating each coefficient of the polynomial to zero yields system of algebraic equations for $a_{0}, a_{1}, \ldots, a_{n}, k$, and $\omega$.

Step 5. By solving the system obtained in Step 4, the constants $a_{0}, a_{1}, \ldots, a_{n}, k$, and $\omega$ can be expressed by the parameters $A$, $B$, and $C$. Depending on the chosen values of $A, B$, and $C$ the function $F(\xi)$ possesses the traveling wave solutions as given above, then the fractional mapping method (8) has the traveling wave solution of the nonlinear FDE (6).

\section{The Space-Time Fractional Combined KdV-mKdV Equation}

The Korteweg-de Vries (KdV) equation, together with its extensions, has been used to describe the balance between nonlinear wave steepening and linear wave dispersion. Also, it is used as the model for nonlinear long waves in different dynamical contexts, including the ion acoustic waves in plasmas, shallow water waves in channels and oceans, pulse waves in large arteries, and surface waves in nonlinear lattice [32]. On the other hand, as an extended form of the KdV equation, the modified $\mathrm{KdV}$ ( $\mathrm{mKdV}$ ) equation has been applied to describe the electromagnetic waves in size-quantized films, Alfvén waves in collision less plasmas, interfacial waves in two-layer liquids, and transmission lines in the Schottky barrier. For certain environmental conditions, when the quadratic nonlinear term is small, the cubic nonlinear term becomes the major one and should be taken into account. The corresponding equation is the space-time fractional combined $\mathrm{KdV}-\mathrm{mKdV}$ equation, for the internal solitary waves in shallow seas and atmosphere dust-acoustic solitary waves and ion acoustic waves in plasmas with negative ions:

$$
D_{t}^{\alpha} u+\mu u D_{x}^{\alpha} u+\delta u^{2} D_{x}^{\alpha} u+\tau D_{x}^{3 \alpha} u=0, \quad 0<\alpha \leq 1,
$$

where $\mu, \delta$, and $\tau$ are constants, which is a transformed generalization of the combined $\mathrm{KdV}-\mathrm{mKdV}$ equation [3335]. In order to solve (17) by the fractional mapping method, we use the traveling wave transformation $u(x, t)=u(\xi)$, $\xi=k x+\omega t$, then, (17) is reduced to the following nonlinear FODE:

$$
\omega^{\alpha} D_{\xi}^{\alpha} u+\mu k^{\alpha} u D_{\xi}^{\alpha} u+\delta k^{\alpha} u^{2} D_{\xi}^{\alpha} u+\tau k^{3 \alpha} D_{\xi}^{3 \alpha} u=0 .
$$

Balancing $D_{\xi}^{3 \alpha} u$ with $u^{2} D_{\xi}^{\alpha} u$ gives $n=1$. Therefore, the solution of (18) can be expressed as

$$
u=a_{0}+a_{1} F .
$$

Substituting (19) into (18) using (10) and setting the coefficients of $F$ to zero, we get

$$
\begin{gathered}
a_{0}=-\frac{\mu}{2 \delta}, \quad a_{1}= \pm k^{\alpha} \sqrt{-\frac{6 \tau C}{\delta}}, \\
\omega=k\left(\frac{\mu^{2}}{4 \delta^{2}}-\tau k^{2 \alpha} B\right)^{1 / \alpha} .
\end{gathered}
$$

The general formula for the travelling wave solution of the space-time fractional combined $\mathrm{KdV}$-mKdV equation (17) is

$$
\begin{gathered}
u=-\frac{\mu}{2 \delta} \pm k^{\alpha} \sqrt{-\frac{6 \tau C}{\delta}} F(k x+\omega t), \\
\omega=k\left(\frac{\mu^{2}}{4 \delta^{2}}-\tau k^{2 \alpha} B\right)^{1 / \alpha} .
\end{gathered}
$$

By selecting the special value of $A, B, C$, and the corresponding function $F(\xi)$, we get the following solutions of (17):

$$
\begin{gathered}
u_{1}=-\frac{\mu}{2 \delta} \pm k^{\alpha} \sqrt{-\frac{6 \tau}{\delta}} \tanh (k x+\omega t, \alpha), \\
\omega=k\left(\frac{\mu^{2}}{4 \delta^{2}}+2 \tau k^{2 \alpha}\right)^{1 / \alpha}, \\
u_{2}=-\frac{\mu}{2 \delta} \pm k^{\alpha} \sqrt{-\frac{6 \tau}{\delta}} \operatorname{coth}(k x+\omega t, \alpha), \\
\omega=k\left(\frac{\mu^{2}}{4 \delta^{2}}+2 \tau k^{2 \alpha}\right)^{1 / \alpha},
\end{gathered}
$$




$$
\begin{gathered}
u_{3}=-\frac{\mu}{2 \delta} \pm k^{\alpha} \sqrt{\frac{6 \tau}{\delta}} \operatorname{sech}(k x+\omega t, \alpha), \\
\omega=k\left(\frac{\mu^{2}}{4 \delta^{2}}-\tau k^{2 \alpha}\right)^{1 / \alpha}, \\
u_{4}=-\frac{\mu}{2 \delta} \pm k^{\alpha} \sqrt{-\frac{6 \tau}{\delta}} \operatorname{csch}(k x+\omega t, \alpha), \\
\omega=k\left(\frac{\mu^{2}}{4 \delta^{2}}-\tau k^{2 \alpha}\right)^{1 / \alpha}, \\
u_{5}=-\frac{\mu}{2 \delta} \pm \frac{k^{\alpha}}{2} \sqrt{-\frac{6 \tau}{\delta}}\left[\frac{\tanh (k x+\omega t, \alpha)}{1 \pm \operatorname{sech}(k x+\omega t, \alpha)}\right] \\
\omega=k\left(\frac{\mu^{2}}{4 \delta^{2}}+\frac{\tau k^{2 \alpha}}{2}\right)^{1 / \alpha} .
\end{gathered}
$$

The remaining solutions can be obtained in a similar manner. With the best of our knowledge, the solutions (24) and (26) are presented for the first time. Also, we can obtain the same solutions (22) and (23) from the fractional Riccati expansion method [25]. When $\alpha=1$, we obtain the classical combined $\mathrm{KdV}-\mathrm{mKdV}$ equation

$$
u_{t}+\mu u u_{x}+\delta u^{2} u_{x}+\tau u_{x x x}=0
$$

as special case of (17). Solutions given in (22)-(26) reduced to the well known solutions of the combined KdV-mKdV equation

$$
\begin{aligned}
& u_{1 \mathrm{ckdv}-\mathrm{mkdv}}=-\frac{\mu}{2 \delta} \pm k \sqrt{-\frac{6 \tau}{\delta}} \tanh (k x+\omega t), \\
& \omega=k\left(\frac{\mu^{2}}{4 \delta^{2}}+2 \tau k^{2}\right) \text {, } \\
& u_{2 \mathrm{ckdv}-\mathrm{mkdv}}=-\frac{\mu}{2 \delta} \pm k \sqrt{-\frac{6 \tau}{\delta}} \operatorname{coth}(k x+\omega t) \text {, } \\
& \omega=k\left(\frac{\mu^{2}}{4 \delta^{2}}+2 \tau k^{2}\right) \text {, } \\
& u_{3 \mathrm{ckdv}-\mathrm{mkdv}}=-\frac{\mu}{2 \delta} \pm k \sqrt{\frac{6 \tau}{\delta}} \operatorname{sech}(k x+\omega t), \\
& \omega=k\left(\frac{\mu^{2}}{4 \delta^{2}}-\tau k^{2}\right) \text {, } \\
& u_{4 \mathrm{ckdv}-\mathrm{mkdv}}=-\frac{\mu}{2 \delta} \pm k \sqrt{-\frac{6 \tau}{\delta}} \operatorname{csch}(k x+\omega t) \text {, } \\
& \omega=k\left(\frac{\mu^{2}}{4 \delta^{2}}-\tau k^{2}\right) \text {, } \\
& u_{5 \mathrm{ckdv}-\mathrm{mkdv}}=-\frac{\mu}{2 \delta} \pm \frac{k}{2} \sqrt{-\frac{6 \tau}{\delta}}\left[\frac{\tanh (k x+\omega t)}{1 \pm \operatorname{sech}(k x+\omega t)}\right] \text {, } \\
& \omega=k\left(\frac{\mu^{2}}{4 \delta^{2}}+\frac{\tau k^{2}}{2}\right) \text {. }
\end{aligned}
$$

\section{Conclusions and Discussions}

In this paper, to construct exact analytical solutions of nonlinear FDEs, the fractional mapping method is presented. The space-time fractional combined $\mathrm{KdV}-\mathrm{mKdV}$ equation is chosen to demonstrate the power of the method. Many exact solutions of the space-time fractional combined $\mathrm{KdV}$ $\mathrm{mKdV}$ equation are derived; these include the generalized hyperbolic and trigonometric function solutions. To the best of our knowledge, some of the solutions obtained in this research paper have not been reported in literature. Mathematical packages can be used to perform more complicated and tedious algebraic calculations. The fractional mapping method can be applied to other nonlinear FDEs. How to extend other methods used for solving differential equations such as Fan subequation method, auxiliary subequation method, and the projective Riccati equation method to handle FDEs is worthy to study. This is our task in the future.

\section{Appendix}

The product of two power series are given by

$$
\left(\sum_{n=0}^{\infty} a_{n} x^{n}\right)\left(\sum_{n=0}^{\infty} b_{n} x^{n}\right)=\sum_{n=0}^{\infty} c_{n} x^{n},
$$

where $c_{n}=\sum_{k=0}^{\infty} a_{k} b_{n-k}$. If $n$ is a natural number, then

$$
\left(\sum_{k=0}^{\infty} a_{k} x^{k}\right)^{n}=\sum_{k=0}^{\infty} c_{k} x^{k},
$$

where $c_{o}=a_{0}^{n}$ and $c_{m}=\left(1 / m a_{0}\right) \sum_{k=1}^{\infty}(k n-m+k) a_{k} c_{m-k}[36]$. For simplicity, we suppose that

$$
\begin{aligned}
E_{\alpha}\left(x^{\alpha}\right) E_{\alpha}\left(-x^{\alpha}\right) & =\left(\sum_{k=0}^{\infty} \frac{x^{\alpha k}}{\Gamma(1+\alpha k)}\right)\left(\sum_{k=0}^{\infty} \frac{\left(-x^{\alpha}\right)^{k}}{\Gamma(1+\alpha k)}\right) \\
& =M .
\end{aligned}
$$

From the definition of $\cosh (x, \alpha)$ and $\sinh (x, \alpha)$, we can get the following inequality:

$$
\begin{aligned}
& \cosh ^{2}(x, \alpha)-\sinh ^{2}(x, \alpha) \\
& =\frac{1}{4}\left[E_{\alpha}\left(x^{\alpha}\right)^{2}+2 E_{\alpha}\left(x^{\alpha}\right) E_{\alpha}\left(-x^{\alpha}\right)\right. \\
& \quad+E_{\alpha}\left(-x^{\alpha}\right)^{2}-E_{\alpha}\left(x^{\alpha}\right)^{2} \\
& \left.\quad+2 E_{\alpha}\left(x^{\alpha}\right) E_{\alpha}\left(-x^{\alpha}\right)-E_{\alpha}\left(-x^{\alpha}\right)^{2}\right] \\
& =E_{\alpha}\left(x^{\alpha}\right) E_{\alpha}\left(-x^{\alpha}\right)=M .
\end{aligned}
$$

Dividing by $\cosh ^{2}(x, \alpha)$ and $\sinh ^{2}(x, \alpha)$, we have

$$
\begin{aligned}
& 1-\tanh ^{2}(x, \alpha)=M \operatorname{sech}^{2}(x, \alpha), \\
& \operatorname{coth}^{2}(x, \alpha)-1=M \operatorname{csch}^{2}(x, \alpha) .
\end{aligned}
$$


Similarly, we suppose that

$$
\begin{gathered}
E_{\alpha}\left(i x^{\alpha}\right) E_{\alpha}\left(-i x^{\alpha}\right)=\left(\sum_{k=0}^{\infty} \frac{\left(i x^{\alpha}\right)^{k}}{\Gamma(1+\alpha k)}\right)\left(\sum_{k=0}^{\infty} \frac{\left(-i x^{\alpha}\right)^{k}}{\Gamma(1+\alpha k)}\right) \\
=\widetilde{M} \\
\cos ^{2}(x, \alpha)+\sin ^{2}(x, \alpha)=E_{\alpha}\left(i x^{\alpha}\right) E_{\alpha}\left(-i x^{\alpha}\right)=\widetilde{M} \\
1+\tan ^{2}(x, \alpha)=\widetilde{M} \sec ^{2}(x, \alpha), \\
\cot ^{2}(x, \alpha)+1=\widetilde{M} \csc ^{2}(x, \alpha) .
\end{gathered}
$$

The fractional derivatives of the Mittag-Leffler function take the form

$$
\begin{aligned}
& D_{x}^{\alpha} E_{\alpha}\left(x^{\alpha}\right)=\sum_{k=0}^{\infty} \frac{D_{x}^{\alpha} x^{\alpha k}}{\Gamma(1+\alpha k)} \\
& =\sum_{k=1}^{\infty} \frac{\Gamma(1+\alpha k) x^{\alpha k-\alpha}}{\Gamma(1+\alpha k) \Gamma(\alpha k+1-\alpha)} \\
& =\sum_{k=1}^{\infty} \frac{x^{\alpha(k-1)}}{\Gamma(\alpha(k-1)+1)} \\
& =\sum_{s=0}^{\infty} \frac{x^{\alpha s}}{\Gamma(\alpha s+1)} \\
& =E_{\alpha}\left(x^{\alpha}\right) \text {, } \\
& D_{x}^{\alpha} E_{\alpha}\left(-x^{\alpha}\right)=\sum_{k=0}^{\infty} \frac{(-1)^{k} D_{x}^{\alpha} x^{\alpha k}}{\Gamma(1+\alpha k)} \\
& =\sum_{k=1}^{\infty} \frac{(-1)^{k} \Gamma(1+\alpha k) x^{\alpha k-\alpha}}{\Gamma(1+\alpha k) \Gamma(\alpha k+1-\alpha)} \\
& =\sum_{k=1}^{\infty} \frac{(-1)^{k} x^{\alpha(k-1)}}{\Gamma(\alpha(k-1)+1)} \\
& =\sum_{s=0}^{\infty} \frac{(-1)^{s+1} x^{\alpha s}}{\Gamma(\alpha s+1)} \\
& =-E_{\alpha}\left(-x^{\alpha}\right), \\
& D_{x}^{\alpha} E_{\alpha}\left(i x^{\alpha}\right)=i E_{\alpha}\left(i x^{\alpha}\right) \text {, } \\
& D_{x}^{\alpha} E_{\alpha}\left(-i x^{\alpha}\right)=-i E_{\alpha}\left(-i x^{\alpha}\right) \text {. }
\end{aligned}
$$

From ((A.7)) and ((A.8)), we can get the derivatives of the generalized hyperbolic functions

$$
\begin{aligned}
D_{x}^{\alpha}[\sinh (x, \alpha)] & =\frac{D_{x}^{\alpha}\left[E_{\alpha}\left(x^{\alpha}\right)\right]-D_{x}^{\alpha}\left[E_{\alpha}\left(-x^{\alpha}\right)\right]}{2} \\
& =\frac{E_{\alpha}\left(x^{\alpha}\right)+E_{\alpha}\left(-x^{\alpha}\right)}{2}=\cosh (x, \alpha),
\end{aligned}
$$

$$
\begin{aligned}
D_{x}^{\alpha}[\cosh (x, \alpha)] & =\frac{D_{x}^{\alpha}\left[E_{\alpha}\left(x^{\alpha}\right)\right]+D_{x}^{\alpha}\left[E_{\alpha}\left(-x^{\alpha}\right)\right]}{2} \\
& =\frac{E_{\alpha}\left(x^{\alpha}\right)-E_{\alpha}\left(-x^{\alpha}\right)}{2}=\sinh (x, \alpha), \\
D_{x}^{\alpha}[\sin (x, \alpha)] & =\frac{D_{x}^{\alpha}\left[E_{\alpha}\left(i x^{\alpha}\right)\right]-D_{x}^{\alpha}\left[E_{\alpha}\left(-i x^{\alpha}\right)\right]}{2 i} \\
& =\frac{E_{\alpha}\left(i x^{\alpha}\right)+E_{\alpha}\left(-i x^{\alpha}\right)}{2}=\cos (x, \alpha), \\
D_{x}^{\alpha}[\cos (x, \alpha)] & =\frac{D_{x}^{\alpha}\left[E_{\alpha}\left(i x^{\alpha}\right)\right]+D_{x}^{\alpha}\left[E_{\alpha}\left(-i x^{\alpha}\right)\right]}{2} \\
& =-\frac{E_{\alpha}\left(x^{\alpha}\right)-E_{\alpha}\left(-x^{\alpha}\right)}{2 i}=-\sin (x, \alpha) .
\end{aligned}
$$

By using these inequalities, we proof the nine cases

Case A.1. If $A=1, B=-2$, and $C=1$, then (10) has the solution $F=\tanh (x, \alpha)$.

$$
\begin{aligned}
\text { L.H.S }= & D_{x}^{\alpha} \tanh (x, \alpha)=D_{x}^{\alpha}\left[\frac{\sinh (x, \alpha)}{\cosh (x, \alpha)}\right] \\
= & D_{x}^{\alpha}\left[\cosh ^{-1}(x, \alpha) \sinh (x, \alpha)\right] \\
= & \cosh ^{-1}(x, \alpha) D_{x}^{\alpha}[\sinh (x, \alpha)] \\
& +\sinh (x, \alpha) D_{x}^{\alpha}\left[\cosh ^{-1}(x, \alpha)\right] \\
= & \cosh ^{-1}(x, \alpha) D_{x}^{\alpha}[\sinh (x, \alpha)] \\
& -\sinh ^{2}(x, \alpha) \cosh ^{-2}(x, \alpha) D_{x}^{\alpha}[\cosh (x, \alpha)] \\
= & \frac{\cosh ^{2}(x, \alpha)-\sinh ^{2}(x, \alpha)}{\cosh ^{2}(x, \alpha)} \\
= & \frac{M}{\cosh ^{2}(x, \alpha)}=M \operatorname{sech}^{2}(x, \alpha), \\
\text { R.H.S }= & {\left[1-2 \tanh ^{2}(x, \alpha)+\tanh ^{4}(x, \alpha)\right]^{1 / 2} } \\
= & 1-\tanh ^{2}(x, \alpha)=M \operatorname{sech}^{2}(x, \alpha) .
\end{aligned}
$$

Then, the two sides are equal. By the same manner, the other formulas can be derived.

\section{Conflict of Interests}

The authors declare that there is no conflict of interests regarding the publication of this paper.

\section{References}

[1] A. A. Kilbas, H. M. Srivastava, and J. J. Trujillo, Theory and Applications of Fractional Differential Equations, Elsevier, Amsterdam, Netherlands, 2006.

[2] R. Hilfer, Applications of Fractional Calculus in Physics, World Scientific, New Jersey, NJ, USA, 2000. 
[3] B. J. West, M. Bologna, and P. Grigolini, Physics of Fractal Operators, Springer, New York, NY, USA, 2003.

[4] K. S. Miller and B. Ross, An Introduction to the Fractional Calculus and Fractional Differential Equations, John Wiley \& Sons, New York, NY, USA, 1993.

[5] S. G. Samko, A. A. Kilbas, and O. I. Marichev, Fractional Integrals and Derivatives: Theory and Applications, Gordon and Breach Science, Yverdon, Switzerland, 1993.

[6] I. Podlubny, Fractional Differential Equations, Academic Press, San Diego, Calif, USA, 1999.

[7] K. Diethelm, The Analysis of Fractional Differential Equations, Springer, Berlin, Germany, 2010.

[8] M. Cui, "Compact finite difference method for the fractional diffusion equation," Journal of Computational Physics, vol. 228, no. 20, pp. 7792-7804, 2009.

[9] X. Zhao and Z. Z. Sun, "Compact Crank-Nicolson schemes for a class of fractional Cattaneo equation in inhomogeneous medium," Journal of Scientific Computing, 2014.

[10] Q. Huang, G. Huang, and H. Zhan, "A finite element solution for the fractional advection-dispersion equation," Advances in Water Resources, vol. 31, no. 12, pp. 1578-1589, 2008.

[11] A. M. A. El-Sayed and M. Gaber, “The Adomian decomposition method for solving partial differential equations of fractal order in finite domains," Physics Letters A, vol. 359, no. 3, pp. 175-182, 2006.

[12] A. M. A. El-Sayed, S. H. Behiry, and W. E. Raslan, "Adomian's decomposition method for solving an intermediate fractional advection-dispersion equation," Computers \& Mathematics with Applications, vol. 59, no. 5, pp. 1759-1765, 2010.

[13] Z. Odibat and S. Momani, "A generalized differential transform method for linear partial differential equations of fractional order," Applied Mathematics Letters, vol. 21, no. 2, pp. 194-199, 2008.

[14] J. H. He, "Variational iteration method for delay differential equations," Communications in Nonlinear Science and Numerical Simulation, vol. 2, no. 4, pp. 235-236, 1997.

[15] G. Wu and E. W. M. Lee, "Fractional variational iteration method and its application," Physics Letters A, vol. 374, no. 25, pp. 2506-2509, 2010.

[16] S. Guo and L. Mei, "The fractional variational iteration method using He's polynomials," Physics Letters A, vol. 375, no. 3, pp. 309-313, 2011.

[17] J. H. He, "Homotopy perturbation technique," Computer Methods in Applied Mechanics and Engineering, vol. 178, no. 3-4, pp. 257-262, 1999.

[18] J. H. He, "A coupling method of a homotopy technique and a perturbation technique for non-linear problems," International Journal of Non-Linear Mechanics, vol. 35, no. 1, pp. 37-43, 2000.

[19] Q. Xu and J. S. Hesthaven, "Stable multi-domain spectral penalty methods for fractional partial differential equations," Journal of Computational Physics, vol. 257, pp. 241-258, 2014.

[20] M. Zayernouri and G. E. Karniadakis, "Exponentially accurate spectral and spectral element methods for fractional ODEs," Journal of Computational Physics, vol. 257, pp. 460-480, 2014.

[21] Q. Xu and J. S. Hesthaven, "Discontinuous Galerkin method for fractional convection-diffusion equations," SIAM Journal on Numerical Analysis, vol. 52, no. 1, pp. 405-423, 2014.

[22] G. Pang, W. Chen, and Z. Fu, "Space-fractional advectiondispersion equations by the Kansa method," Journal of Computational Physics, 2014.
[23] S. Zhang and H. Q. Zhang, "Fractional sub-equation method and its applications to nonlinear fractional PDEs," Physics Letters A, vol. 375, no. 7, pp. 1069-1073, 2011.

[24] S. Guo, L. Mei, Y. Li, and Y. Sun, "The improved fractional sub-equation method and its applications to the space-time fractional differential equations in fluid mechanics," Physics Letters A, vol. 376, no. 4, pp. 407-411, 2012.

[25] E. A. Abdel-Salam and E. A. Yousif, "Solution of nonlinear space-time fractional differential equations using the fractional Riccati expansion method," Mathematical Problems in Engineering, vol. 2013, Article ID 846283, 6 pages, 2013.

[26] G. Jumarie, "Modified Riemann-Liouville derivative and fractional Taylor series of nondifferentiable functions further results," Computers \& Mathematics with Applications, vol. 51, no. 9-10, pp. 1367-1376, 2006.

[27] G. Jumarie, "New stochastic fractional models for Malthusian growth, the Poissonian birth process and optimal management of populations," Mathematical and Computer Modelling, vol. 44, no. 3-4, pp. 231-254, 2006.

[28] G. Jumarie, "Laplace's transform of fractional order via the Mittag-Leffler function and modified Riemann-LIOuville derivative," Applied Mathematics Letters, vol. 22, no. 11, pp. 1659-1664, 2009.

[29] R. Almeida and D. F. M. Torres, "Fractional variational calculus for nondifferentiable functions," Computers Mathematics with Applications, vol. 61, pp. 3097-3104, 2011.

[30] K. M. Kolwankar, "Decomposition of Lebesgue-Cantor devil's staircase," Fractals, vol. 12, no. 4, pp. 375-380, 2004.

[31] G. Jumarie, "An approach to differential geometry of fractional order via modified Riemann-Liouville derivative," Acta Mathematica Sinica, vol. 28, no. 9, pp. 1741-1768, 2012.

[32] P. G. Drazin and R. S. Johnson, Solitons : An Introduction, Cambridge University Press, Cambridge, UK, 1989.

[33] A. H. Khater, O. H. El-Kakaawy, and D. K. Callebaut, "Bäcklund transformations and exact solutions for Alfvén solitons in a relativistic electron-positron plasma," Physica Scripta, vol. 58, no. 6 , p. $545,1998$.

[34] T. Kakutani and N. Yamasaki, "Solitary waves on a two-layer fluid," Journal of the Physical Society of Japan, vol. 45, no. 2, pp. 674-679, 1978.

[35] E. A.-B. Abdel-Salam, "Quasi-periodic, periodic waves, and soliton solutions for the combined KdV-mKdV equation," Zeitschrift fur Naturforschung A, vol. 64, no. 9-10, pp. 639-645, 2009.

[36] M. I. Nouh and A. S. Saad, "A new analytical solution to the relativistic polytropic fluid spheres," International Review of Physics, vol. 7, no. 1, pp. 16-21, 2013. 


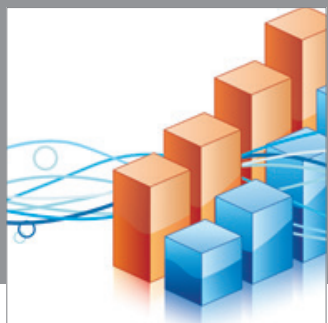

Advances in

Operations Research

mansans

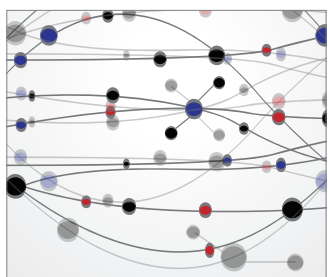

The Scientific World Journal
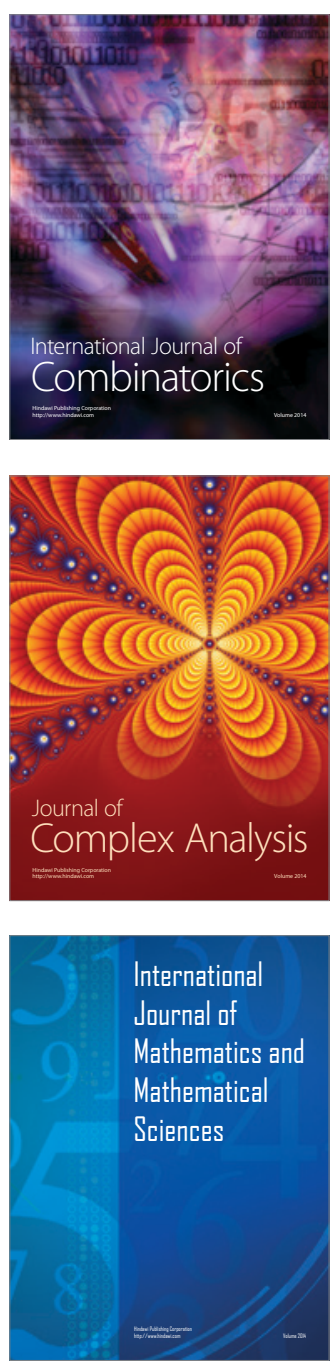
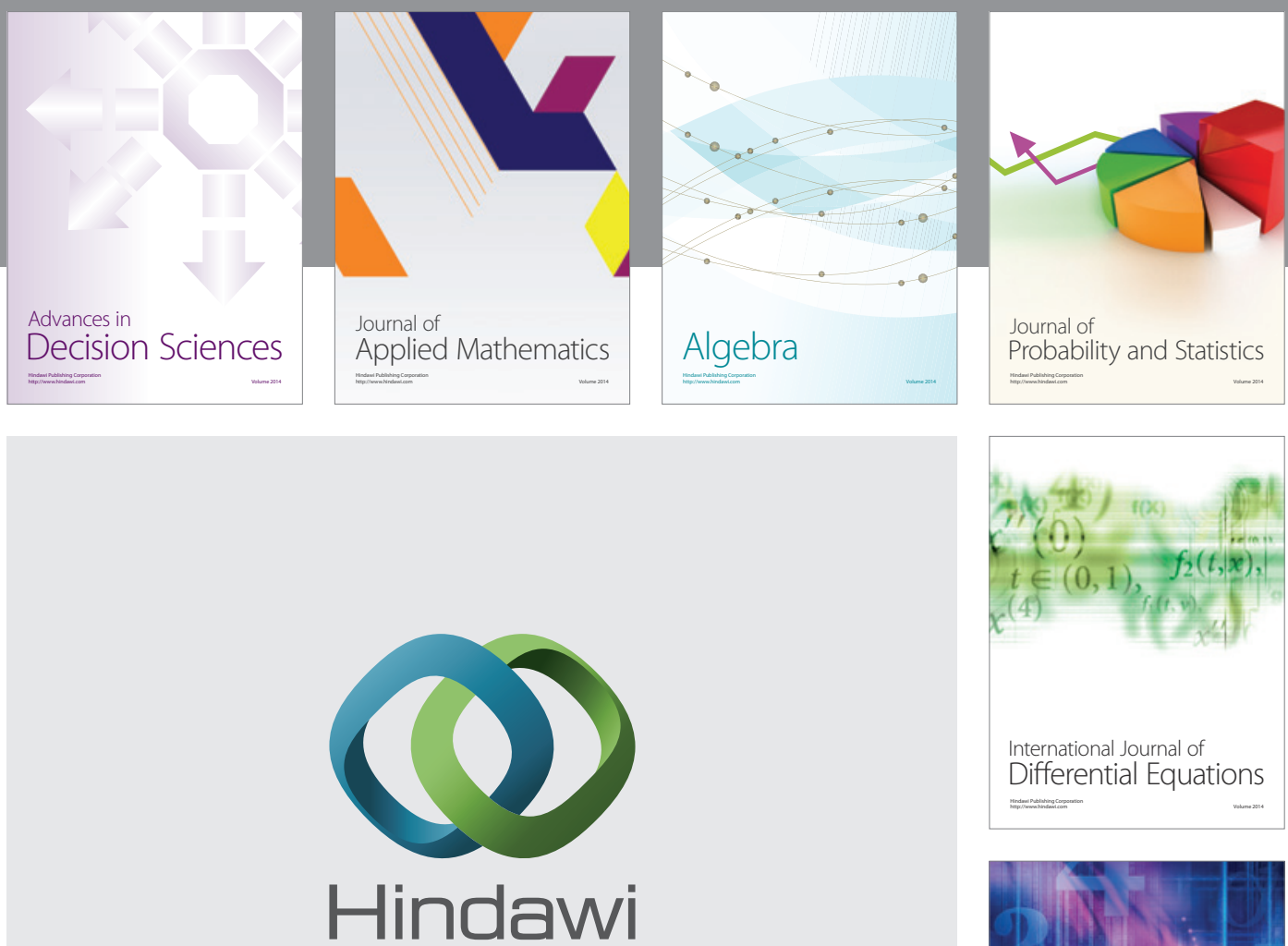

Submit your manuscripts at http://www.hindawi.com
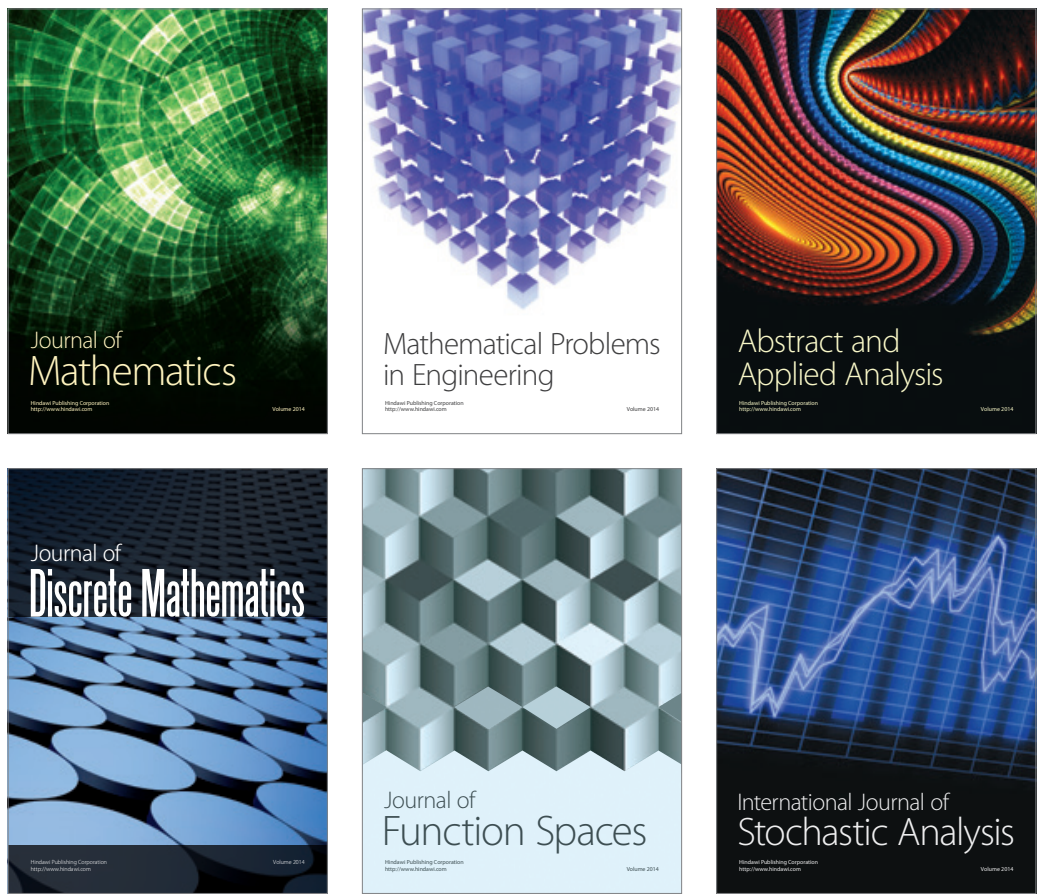

Journal of

Function Spaces

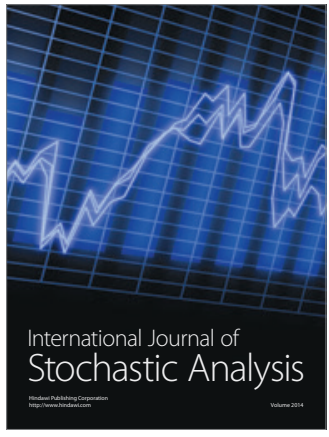

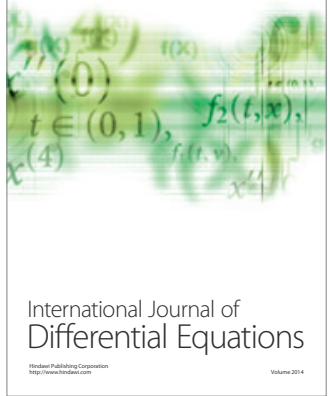
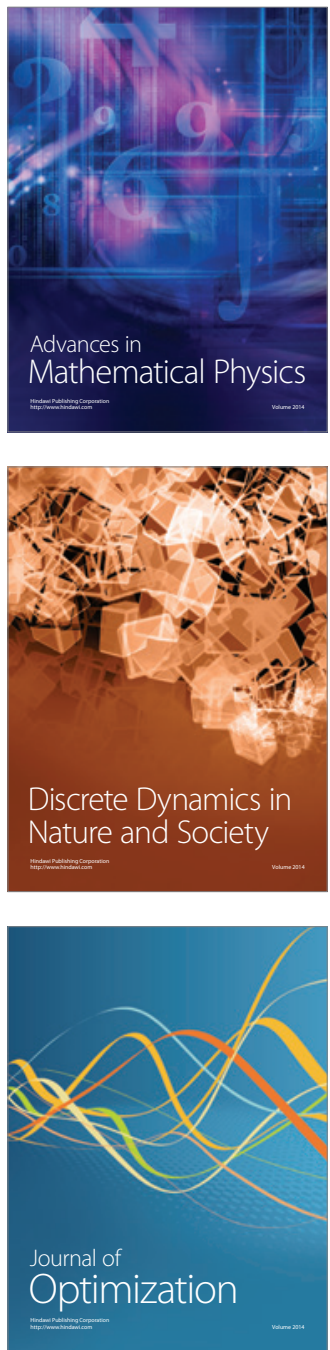